\title{
The Acoustic Pressure Radiated by a Vibrating Circular Plate within the Fraunhofer Zone of the Three-Wall Corner Region
}

\author{
K. Szemela*, W.P. RdZAnek And W.J. RdzAneK
}

Department of Acoustics, Institute of Physics, University of Rzeszów, T. Rejtana 16A, 35-310 Rzeszów, Poland

\begin{abstract}
The Neumann boundary value problem has been solved for the region bounded by the three perfect rigid infinite baffles arranged perpendicularly to one another. The harmonically vibrating clamped circular plate embedded in one of the baffles is the sound source. It has been assumed that the amplitude of the plate's transverse vibrations is small to use the linear Kelvin-Voigt theory. The Green function has been applied to obtain the asymptotic formulae describing the distribution of the acoustic pressure within the Fraunhofer zone. The analysis of sound radiation has been performed for some selected surface excitations and for some different plate's locations. The acoustic pressure distribution has been examined including the acoustic attenuation and the internal attenuation of the plate's material.
\end{abstract}

PACS: 43.20.Ks, 43.20.Rz, 43.40.+s, 43.20.-f, 43.20.+g

\section{Introduction}

Some vibrating flat surfaces generate the acoustic waves which are very often radiated within the regions limited by some kinds of baffles. The baffles reflect the acoustic waves and influence the acoustic pressure distribution within the region. Additionally, this effect modifies the source vibrations and its acoustic properties as well. The baffles' influence on the sound radiation is particularly significant when the acoustic radiator is embedded in one of the baffles and its distance from the remaining baffles is small compared with the acoustic wavelength.

From a practical point of view, it is very important to investigate the acoustic source radiation within the regions limited by the flat baffles arranged perpendicularly to one another. These regions are often found in many building constructions such as industrial halls and residential buildings. Some walls of buildings can be considered as such baffles on certain conditions. The analysis of the sound radiation for such regions allows determining, e.g. the location of the field point at which the acoustic pressure amplitude has the greatest value and the best source location for noise minimizing.

The results obtained on the basis of theoretical investigations can be used for predictions of the properties of acoustic sources and consequently for the noise reduction. However, the pure theoretical analysis of the sound radiation is possible when some simplifying assumptions are introduced, e.g. that the baffles are perfectly rigid and their dimensions are much larger than the acoustic wavelength. In this case, the Neumann boundary value problem has been obtained. The acoustic pressure is often formulated as the double infinite series of the modal components defined by the formulae containing double

\footnotetext{
* corresponding author; e-mail: alpha@univ.rzeszow.pl
}

integrals. Due to summation and integration, the acoustic pressure numerical calculations are time-consuming. Therefore, it is important to obtain some approximate formulae in their elementary forms which enable to simplify the numerical calculation and perform a wide analysis of the acoustic source properties. There are many practical cases when it is only necessary to find the acoustic pressure distribution at large distance from the sound source.

The far field approximation can be used to simplify the formulae for the acoustic pressure. The acoustic attenuation influences significantly the sound radiation and this factor should be included. However, this requires calculating the modal components of acoustic impedance and causes some numerical difficulties, especially, when the large number of vibrating modes is used. An error occurs when the acoustic attenuation is neglected. The value of this error depends on the vibration frequency and can be large for some cases. Therefore, it is very important to perform the numerical analysis of the error. It allows to indicate some frequencies for which the error values are acceptably small and the acoustic attenuation can be neglected. The analysis allows determining the frequency range within the error achieves some large values and the acoustic attenuation has to be included.

So far, the acoustic radiation of some surface sound sources located on a flat baffle was the main aim of the theoretical as well as experimental studies. The acoustic pressure distribution and the energy aspect of the sound radiation have been often analyzed [1-10]. The influence of the point force excitation on the acoustic radiation of double-leaf plate has been determined in [11] where the theoretical as well as experimental approach has been used. The radiation efficiency for some vibrating plates of different shapes has been obtained based on the discrete calculation method [12]. The results presented are valid for the low frequencies. The piezoelectric effect has also 
been used for the active sound reduction in some cases [13-17].

There are only few studies focused on the sound radiation within the region bounded by more than one flat rigid infinite baffle. The simplest regions of this kind are: the two-wall corner - quarter-space bounded by two baffles arranged perpendicularly to one another and the three-wall corner — region bounded by three baffles arranged perpendicularly to one another. The sound radiation of the vibrating sphere in the vicinity of the quarter-space has been investigated in [18]. The total power has been obtained for the two pistons system in the case when the sound sources are located on the two different walls of three-wall corner region [19]. The acoustic pressure distributions within the two and three-wall corner regions have been analyzed for the circular piston located on one of the baffles [20]. The sound radiation within the two-wall corner region has also been investigated for the more complex source such as vibrating membrane of deformable surface. Assuming that the membrane is located on one of the baffles, the total acoustic power has been analyzed [21]. Moreover, the asymptotic expressions for the acoustic pressure distribution have been obtained [22]. They are valid within the Fraunhofer zone. The total acoustics power has been analyzed in the case of the clamped circular plate embedded on the boundary of three-wall corner region [23]. The analysis has been performed for some different plate locations and for some selected external excitations.

The acoustic pressure has not yet been analyzed in the case of the plate located on the boundary of three-wall corner region. Therefore, the main aim of this study is to obtain the asymptotic formulae valid within the Fraunhofer zone to describe the acoustic pressure distribution radiated by the clamped circular plate. Moreover, making use of the results obtained in [23] enables including the acoustic attenuation. The formulae necessary for that purpose have been presented in Appendix for the readers' convenience. The obtained asymptotic formulae of the acoustic pressure have the elementary form and have been used to perform analysis of the acoustic pressure for the two selected surface excitations and for some sample plate's locations. The error resulting from the negligence of the acoustic attenuation has also been investigated.

\section{The analysis assumptions}

The analysis of the acoustic pressure distribution has been performed into the region bounded by infinite baffles arranged perpendicularly to one another. The global Cartesian coordinate system has been introduced to describe an analyzed phenomenon. The origin of this coordinate system is located at the common point of the all baffles and constitutes the centre point of the three-wall corner region. The axes of the coordinate system are determined by the points of the baffles intersections. The considered three-wall corner region can be mathematically defined as $\Omega=\{0 \leq x<\infty, 0 \leq y<\infty$, $0 \leq z<\infty\}$. The sound source is a vibrating clamped circular plate embedded in the baffle located on the plane

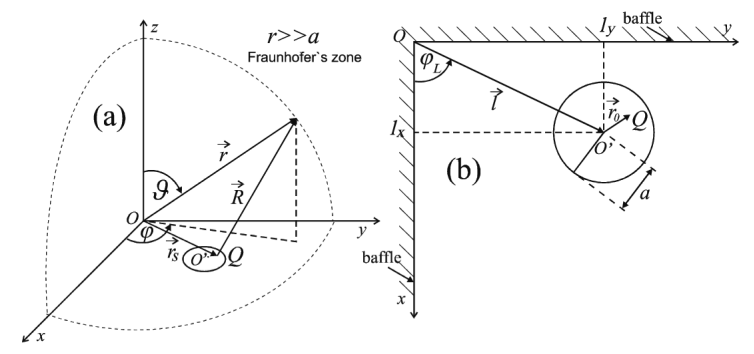

Fig. 1. The vibrating plate at the boundary of three-wall corner region: (a) the Fraunhofer zone, the leading vector of the field point $\boldsymbol{r}$, the leading vector of the source point $\boldsymbol{r}_{s}$, the field point $P$, the source point $Q$, the spherical coordinates $r=|\boldsymbol{r}|, \vartheta, \varphi$; (b) the location of source and the vertical baffles in the global Cartesian coordinates system, the vector of the source point $\boldsymbol{r}_{0}$ in the local coordinates system.

$z=0$ (see Fig. 1). The considered plate is made of the homogeneous and isotropic material of density $\rho$. The plate's radius equals $a$. It has been assumed that the plate's thickness $h$ is much smaller than its radius which enables to use the theory of thin plates. The plate centre is indicated by the vector $\boldsymbol{l}=\left[l_{x}, l_{y}, 0\right]$. The field point $P$ location is described by the leading vector $\boldsymbol{r}=[x, y, z]$. The coordinates of the source point $Q$ are determined by the vector $\boldsymbol{r}_{s}=\left[x_{s}, y_{s}, 0\right]$. Additionally, the local polar coordinate system $\left[r_{0}, \varphi_{0}\right]$ has been introduced to describe the source points location. The origin of the coordinate system is located at the plate centre and its radial axis is parallel to $x$ axis. The relative location of the source point $Q$ and the field point $P$ is determined by the vector $\boldsymbol{R}$. It has been assumed that the region $\Omega$ is filled by the lossless light fluid medium of density $\rho_{0}$. The sound velocity equals $c$. There is interaction between the plate's vibrating surface and the medium which is inside the considered region. The baffles of the three-wall corner region are perfect rigid and perfectly reflect acoustic waves. There is a vacuum outside the region $\Omega$. The vertical baffles cause that the plate's vibrations are asymmetric. The external asymmetric excitation is applied to the plate's surface. The analyzed processes are state and time-harmonic. They vary with respect to time according to relation $\exp (-\mathrm{i} \omega t)$, where $\mathrm{i}^{2}=-1$ and $\omega$ is the excitation circular frequency. The acoustic pressure amplitude has been analyzed at the field points located at large distance from the origin of Cartesian coordinate system when $|\boldsymbol{r}| \gg a$. It has been assumed that the amplitude of the plate's transverse vibrations is small and the plate's motion can be described by the linear model. Using the Kelvin-Voigt theory the equation of motion can be formulated as

$$
\begin{aligned}
& \left(k_{T}^{-4} \nabla^{4}-1\right) v\left(r_{0}, \varphi_{0}\right)-\mathrm{i} \frac{p\left(r_{0}, \varphi_{0}\right)}{\rho h \omega} \\
& \quad=-\mathrm{i} \frac{p^{\text {(excit. })}\left(r_{0}, \varphi_{0}\right)}{\rho h \omega}
\end{aligned}
$$


where

$$
k_{T}^{-4}=\frac{D_{T}}{\rho h \omega^{2}}, \quad D_{T}=D_{E}(1-i \omega \bar{\eta})
$$

$v\left(r_{0}, \varphi_{0}\right)$ is the velocity amplitude of the plate's transverse vibrations, $p\left(r_{0}, \varphi_{0}\right)$ is the amplitude of the acoustic pressure exerted on the plate's surface by the medium, $p^{\text {(excit.) }}\left(r_{0}, \varphi_{0}\right)$ is the amplitude of the external excitation, $D_{E}=E h^{3} /\left[12\left(1-\nu^{2}\right)\right], E, \nu$ denote Young's modulus and the Poisson ratio, respectively, $\bar{\eta}$ is plate's internal attenuation factor [23, 24]. The formulae which allow to solve Eq. (1) including the acoustic attenuation and some external excitations have been presented in Appendix.

\section{The acoustic pressure within the Fraunhofer zone}

The acoustic pressure can be obtained based on the following formula:

$$
p(\boldsymbol{r})=-\mathrm{i} k_{0} \rho_{0} c \int_{S^{\prime}} v\left(\boldsymbol{r}_{s}\right) G\left(\boldsymbol{r} \mid \boldsymbol{r}_{s}\right) \mathrm{d} S^{\prime},
$$

where $\rho_{0}$ is the medium density, $k=2 \pi / \lambda$ is the acoustic wave number, $\lambda$ is the acoustic wavelength, $c$ is the sound velocity in the medium, $v\left(\boldsymbol{r}_{s}\right)$ is the normal component of the vibration velocity amplitude, $G\left(\boldsymbol{r} \mid \boldsymbol{r}_{s}\right)$ denotes the Green function of the Neumann boundary value problem, $S^{\prime}$ is the surface directly adjacent to the surface of sound source [25]. The acoustic pressure (3) can be presented after using Eq. (1) as the double series of the modal components [23]

$$
p(\boldsymbol{r})=\sum_{m=0}^{\infty} \sum_{n=1}^{\infty} \frac{\omega}{\omega_{m, n}}\left[c_{m, n}^{(c)} p_{m, n}^{(c)}(\boldsymbol{r})+c_{m, n}^{(s)} p_{m, n}^{(s)}(\boldsymbol{r})\right],
$$

where

$$
\begin{aligned}
& \left\{\begin{array}{l}
p_{m, n}^{(c)}(\boldsymbol{r}) \\
p_{m, n}^{(s)}(\boldsymbol{r})
\end{array}\right\}=-k_{0} \rho_{0} c \omega_{m, n} \int_{S^{\prime}}\left\{\begin{array}{l}
W_{m, n}^{(c)}\left(r_{0}, \varphi_{0}\right) \\
W_{m, n}^{(s)}\left(r_{0}, \varphi_{0}\right)
\end{array}\right\} \\
& \quad \times G\left(\boldsymbol{r} \mid \boldsymbol{r}_{s}\right) \mathrm{d} S^{\prime} .
\end{aligned}
$$

The Green function for the analyzed problem can be expressed on the basis of the Weyl equation [20, 26]:

$$
\begin{gathered}
G\left(\boldsymbol{r} \mid \boldsymbol{r}_{s}\right)=G_{l_{x}, l_{y}}\left(\boldsymbol{r} \mid \boldsymbol{r}_{s}\right)+G_{-l_{x}, l_{y}}\left(\boldsymbol{r} \mid \boldsymbol{r}_{s}\right) \\
+G_{l_{x},-l_{y}}\left(\boldsymbol{r} \mid \boldsymbol{r}_{s}\right)+G_{-l_{x},-l_{y}}\left(\boldsymbol{r} \mid \boldsymbol{r}_{s}\right),
\end{gathered}
$$

where

$$
\begin{aligned}
& G_{u, v}\left(\boldsymbol{r} \mid \boldsymbol{r}_{s}\right)=\frac{\exp \left(\mathrm{i} k_{0} R_{u, v}\right)}{2 \pi R_{u, v}}, \\
& R_{u, v}=\sqrt{\left[x-\operatorname{sgn}(u) x_{s}\right]^{2}+\left[y-\operatorname{sgn}(v) y_{s}\right]^{2}+z^{2}},
\end{aligned}
$$

$\operatorname{sgn}(\cdot)$ denotes the signum function. The presented form of the Green function can also be obtained using the method of images. The first component in Eq. (6) can be interpreted as the Green function of the real source when there are no vertical baffles. The remaining three components represent the Green function for the image sources (see Fig. 2). The quantity $R_{u, v}$ for $u \in\left\{l_{x},-l_{x}\right\}$ and $v \in\left\{l_{y},-l_{y}\right\}$ is a distance between the point located on the surface of one of sources and the field point $P=P(x, y, z)$.

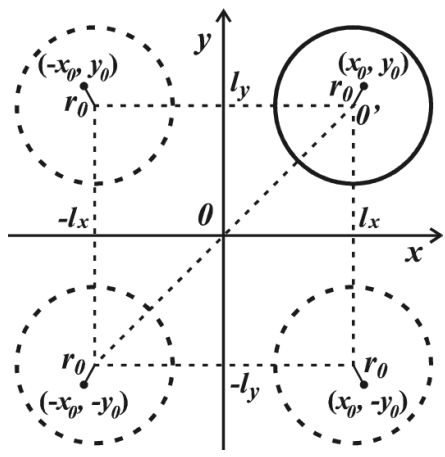

Fig. 2. The location of the real source and the image sources in the global Cartesian coordinates system. Solid line denotes the real source and dashed lines represent the image sources.

The source point's coordinates have been presented by their local counterpairs as follows:

$$
x_{s}=l_{x}+r_{0} \cos \varphi_{0}, \quad y_{s}=l_{y}+r_{0} \sin \varphi_{0} .
$$

Then, the distances $(7 \mathrm{~b})$ have been written in the global spherical coordinate system $[r, \varphi, \vartheta]$ using the following transformations: $x=r \sin \vartheta \cos \varphi, y=r \sin \vartheta \sin \varphi$, $z=r \cos \vartheta$ (cf. [20, 22]):

$$
\begin{aligned}
& \left(\frac{R_{u, v}}{r}\right)^{2}=1+\left(\frac{l_{x}}{r}\right)^{2}+\left(\frac{l_{y}}{r}\right)^{2}+\left(\frac{r_{0}}{r}\right)^{2} \\
& +2 \frac{r_{0}}{r} \frac{l_{x} \cos \varphi_{0}+l_{y} \sin \varphi_{0}}{r} \\
& \quad-2 \sin \vartheta\left[\frac{u \cos \varphi+v \sin \varphi}{r}+\frac{r_{0}}{r}\left(\operatorname{sgn}(u) \cos \varphi \cos \varphi_{0}\right.\right. \\
& \left.\left.+\operatorname{sgn}(v) \sin \varphi \sin \varphi_{0}\right)\right] .
\end{aligned}
$$

The acoustic pressure at large distance from the coordinate system origin can be obtained assuming that $r \gg r_{0}$. The expression $R_{u, v} / r$ has been presented as the limited to the terms of zero and first order expansion series of variable $r_{0} / r$. Finally, the approximation for distance (7b) can be written as

$$
R_{u, v} \approx Q_{u, v}+r_{0}\left(A_{u, v} \cos \varphi_{0}+B_{u, v} \sin \varphi_{0}\right),
$$

where

$$
\begin{aligned}
Q_{u, v} & =\sqrt{r^{2}+l_{x}^{2}+l_{y}^{2}-2 r \sin \vartheta(u \cos \varphi+v \sin \varphi)} \\
A_{u, v} & =\frac{l_{x}-\operatorname{sgn}(u) r \sin \vartheta \cos \varphi}{Q_{u, v}} \\
B_{u, v} & =\frac{l_{y}-\operatorname{sgn}(v) r \sin \vartheta \sin \varphi}{Q_{u, v}}
\end{aligned}
$$

The approximation from Eq. (10) enables to obtain the 
asymptotic formula for the expression given by Eq. (7a) and consequently for the acoustic pressure. The denominator in Eq. (7a) is slowly varying with change of $R_{u, v}$ and can be replaced by only first component from Eq. (10). The approximate formula can be presented in the form of

$$
G_{u, v}\left(\boldsymbol{r} \mid \boldsymbol{r}_{s}\right) \approx \frac{\exp \left(\mathrm{i} k_{0} Q_{u, v}\right)}{2 \pi Q_{u, v}}
$$

$$
\times \exp \left(\mathrm{i} k_{0} r_{0}\left(A_{u, v} \cos \varphi_{0}+B_{u, v} \sin \varphi_{0}\right)\right) .
$$

The modal quantities (5) related to the acoustic pressure can be formulated on the basis of Eqs. (6) and (12) as follows:

$$
\left\{\begin{array}{l}
p_{m, n}^{(c)}(\boldsymbol{r}) \\
p_{m, n}^{(s)}(\boldsymbol{r})
\end{array}\right\} \approx\left\{\begin{array}{l}
\tilde{p}_{m, n}^{(c)}\left(\boldsymbol{r}, l_{x}, l_{y}\right)+\tilde{p}_{m, n}^{(c)}\left(\boldsymbol{r},-l_{x}, l_{y}\right)+\tilde{p}_{m, n}^{(c)}\left(\boldsymbol{r}, l_{x},-l_{y}\right)+\tilde{p}_{m, n}^{(c)}\left(\boldsymbol{r},-l_{x},-l_{y}\right) \\
\tilde{p}_{m, n}^{(s)}\left(\boldsymbol{r}, l_{x}, l_{y}\right)+\tilde{p}_{m, n}^{(s)}\left(\boldsymbol{r},-l_{x}, l_{y}\right)+\tilde{p}_{m, n}^{(s)}\left(\boldsymbol{r}, l_{x},-l_{y}\right)+\tilde{p}_{m, n}^{(s)}\left(\boldsymbol{r},-l_{x},-l_{y}\right)
\end{array}\right\}
$$

where

$$
\begin{aligned}
& \left\{\begin{array}{l}
\tilde{p}_{m, n}^{(c)}(\boldsymbol{r}, u, v) \\
\tilde{p}_{m, n}^{(s)}(\boldsymbol{r}, u, v)
\end{array}\right\}=-k_{0} \rho_{0} c \omega_{m, n} \frac{\exp \left(\mathrm{i} k_{0} Q_{u, v}\right)}{Q_{u, v}} \\
& \quad \times \int_{0}^{a} W_{m, n}\left(r_{0}\right)\left\{\begin{array}{l}
F_{m}^{(c)}\left(r_{0}\right) \\
F_{m}^{(s)}\left(r_{0}\right)
\end{array}\right\} r_{0} \mathrm{~d} r_{0}
\end{aligned}
$$

and

$$
\begin{aligned}
& \left\{\begin{array}{l}
F_{m}^{(c)}\left(r_{0}\right) \\
F_{m}^{(s)}\left(r_{0}\right)
\end{array}\right\}=\frac{1}{2 \pi} \int_{0}^{2 \pi} \exp \left(\mathrm { i } k _ { 0 } r _ { 0 } \left(A_{u, v} \cos \varphi_{0}\right.\right. \\
& \left.\left.+B_{u, v} \sin \varphi_{0}\right)\right)\left\{\begin{array}{l}
\cos m \varphi_{0} \\
\sin m \varphi_{0}
\end{array}\right\} \mathrm{d} \varphi_{0} .
\end{aligned}
$$

In order to perform the integration in Eq. (15) it is necessary to introduce the following substitutions:

$$
k_{0} A_{u, v}=\Lambda / a \cos w, \quad k_{0} B_{u, v}=\Lambda / a \sin w .
$$

Making use of the following formulae:

$$
\begin{gathered}
\int_{0}^{2 \pi} \cos (\bar{a}) \cos (\bar{b})\left\{\begin{array}{l}
\cos m \varphi \\
\sin m \varphi
\end{array}\right\} \mathrm{d} \varphi \\
=2 \pi \cos (m \pi / 2) J_{m}(Z)\left\{\begin{array}{l}
\cos m \alpha \\
0
\end{array}\right. \\
\int_{0}^{2 \pi} \cos (\bar{a}) \sin (\bar{b})\left\{\begin{array}{l}
\cos m \varphi \\
\sin m \varphi
\end{array}\right\} \mathrm{d} \varphi \\
=2 \pi \sin (m \pi / 2) J_{m}(Z)\left\{\begin{array}{l}
0 \\
\sin m \alpha
\end{array}\right. \\
\int_{0}^{2 \pi} \sin (\bar{a}) \cos (\bar{b})\left\{\begin{array}{l}
\cos m \varphi \\
\sin m \varphi
\end{array}\right\} \mathrm{d} \varphi \\
=2 \pi \sin (m \pi / 2) J_{m}(Z)\left\{\begin{array}{l}
\cos m \alpha \\
0
\end{array}\right.
\end{gathered}
$$

$$
\begin{aligned}
& \int_{0}^{2 \pi} \sin (\bar{a}) \sin (\bar{b})\left\{\begin{array}{c}
\cos m \varphi \\
\sin m \varphi
\end{array}\right\} \mathrm{d} \varphi \\
& =-2 \pi \cos (m \pi / 2) J_{m}(Z)\left\{\begin{array}{l}
0 \\
\sin m \alpha
\end{array}\right.
\end{aligned}
$$

where $\bar{a}=Z \cos \alpha \cos \varphi, \bar{b}=Z \sin \alpha \sin \varphi$, leads to

$$
\begin{aligned}
& \left\{\begin{array}{l}
F_{m}^{(c)}\left(r_{0}\right) \\
F_{m}^{(s)}\left(r_{0}\right)
\end{array}\right\}=\exp (\mathrm{i} m \pi / 2) \\
& \times J_{m}\left(\Lambda r_{0} / a\right)\left\{\begin{array}{c}
\cos m w \\
\sin m w
\end{array}\right\} .
\end{aligned}
$$

The values of $\Lambda$ and $w$ should be found from Eqs. (16). Inserting Eq. (17) into Eq. (14) and performing integration over the radial variable yields

$$
\begin{aligned}
& \left\{\begin{array}{l}
\tilde{p}_{m, n}^{(c)}(\boldsymbol{r}, u, v) \\
\tilde{p}_{m, n}^{(s)}(\boldsymbol{r}, u, v)
\end{array}\right\}=-\beta a \rho_{0} c \omega_{m, n} \\
& \quad \times \frac{\exp \left(\mathrm{i} k_{0} Q_{u, v}\right) \sqrt{2 \varepsilon_{m}} \beta_{m, n}^{2}}{Q_{u, v}\left(\beta_{m, n}^{4}-\Lambda^{4}\right)} \\
& \times\left(\beta_{m, n} \alpha_{m, n} J_{m}(\Lambda)-\Lambda J_{m+1}(\Lambda)\right) \\
& \quad \times \exp (\mathrm{i} m \pi / 2)\left\{\begin{array}{c}
\cos m w \\
\sin m w
\end{array}\right\}, \\
& \text { where } \beta=k_{0} a, \alpha_{m, n}=J_{m+1}\left(\beta_{m, n}\right) / J_{m}\left(\beta_{m, n}\right) .
\end{aligned}
$$

After obtaining constants $c_{m, n}^{(c)}, c_{m, n}^{(s)}$ from Eqs. (A.8), the acoustic pressure within the Fraunhofer zone can be calculated including the acoustic attenuation on the basis of the formulae given by Eqs. (4), (13) and (18). The presented expressions can also be used for the acoustic pressure calculations in the special cases i.e. when there are no vertical baffles or when only one vertical baffle exists. The formula (13) limited to the first component is valid when the vertical baffles do not exist. Using only two first components in Eq. (13) leads to the expressions presenting the acoustic pressure in the case when there is only one vertical baffle located on the plane $x z$. The 
numerical analysis of the acoustic pressure distribution within the Fraunhofer zone has been performed based on the obtained formulae. The selected external excitation and some different plate locations have been analyzed.

\section{Numerical analysis}

Based on the obtained asymptotic expressions, the numerical analysis of the distribution of acoustic pressure amplitude has been performed within the Fraunhofer zone of the three-wall corner region. The sound source is the vibrating clamped circular plate asymmetrically excited. The formulae presented in Appendix have been used to investigate the acoustic attenuation influence on the sound radiation. They also enable to include the excitation of any asymmetric surface distribution. The following two arbitrary surface excitations have been selected for the numerical analysis:

$$
p_{1}^{\text {(excit.) }}\left(r_{0}, \varphi_{0}\right)=\frac{F_{0}}{r_{0}} \delta\left(r_{0}-\bar{r}_{0}\right) \delta\left(\varphi_{0}-\bar{\varphi}_{0}\right)
$$

$$
p_{2}^{\text {(excit.) }}\left(r_{0}, \varphi_{0}\right)= \begin{cases}p_{0}^{\text {(excit.) }} ; & \bar{r}_{0}-\Delta \bar{r}_{0} / 2 \leq r_{0} \leq \bar{r}_{0}+\Delta \bar{r}_{0} / 2 \quad \text { and } \quad \bar{\varphi}_{0}-\Delta \bar{\varphi}_{0} / 2 \leq \varphi_{0} \leq \bar{\varphi}_{0}+\Delta \bar{\varphi}_{0} / 2 \\ 0 ; & \text { otherwise }\end{cases}
$$

where $\delta(\cdot)$ is the Dirac delta function, $F_{0}[\mathrm{~N}], p_{0}^{(\text {excit. })}[\mathrm{Pa}]$. The excitation defined by Eq. (19a) is the point excitation representing the concentrated force of the amplitude $F_{0}$ which is exerted at the surface point $\left[\bar{r}_{0}, \bar{\varphi}_{0}\right]$. This is the asymmetric excitation in the mathematical form convenient for further calculations. In particular, when $r_{0}=0$, the excitation is symmetric. Equation (19b) presents the excitation of the pressure amplitude $p_{0}^{\text {(excit.) }}$ applied to the plate's segment of area $S_{0}=\bar{r}_{0} \Delta \bar{r}_{0} \Delta \bar{\varphi}_{0}$. In the case of this excitation, the sound radiation of the vibrating plate has been analyzed in detail in [23]. The centre point of the excited plate's segment is located at the source point whose polar coordinates are equal to $\left[\bar{r}_{0}, \bar{\varphi}_{0}\right]$ (cf. [23]). It has been assumed that $\Delta \bar{r}_{0}=\sqrt{S_{0}}$, $\Delta \bar{\varphi}_{0}=\sqrt{S_{0}} / \bar{r}_{0}$ and $S_{0} \ll S$. The first two conditions mean that the shape of plate's segment becomes similar to a square when its distance from the plate's centre grows. The excitation can be implemented by an electrically controlled piezoelectric element located on the

plate's surface. The shape of the piezoelectric element should be similar to the plate's segment. The excitation models defined by Eqs. (19) has been chosen for some practical reasons. Namely, the excitation from Eq. (19b) can be modeled by the excitation from Eq. (19a) in the case of the low frequencies when the length of the radiated acoustic waves is large in comparison with the geometric dimensions of the excited plate's segment. Moreover, the area of plate's segment should be small compared with the whole plate's area. The use of the simpler mathematical model of the excitation simplifies the numerical calculations.

The expressions from Eq. (A.7) can be formulated in the case of the chosen excitations as

$$
\left\{\begin{array}{l}
P_{1, m, n}^{(\text {excit. }, c)} \\
P_{1, m, n}^{(\text {excit. }, s)}
\end{array}\right\}=F_{0}\left\{\begin{array}{l}
W_{m, n}^{(c)}\left(\bar{r}_{0}, \bar{\varphi}_{0}\right) \\
W_{m, n}^{(s)}\left(\bar{r}_{0}, \bar{\varphi}_{0}\right)
\end{array}\right\}
$$

$$
\begin{aligned}
& P_{2, m, n}^{\text {(excit. }, c)}=\frac{p_{0}^{\text {(excit.) }} \bar{w}(\bar{r})}{S}\left\{\begin{array}{l|l}
\Delta \bar{\varphi}_{0} ; \\
1 / m\left[\sin \left(m\left(\bar{\varphi}_{0}+\Delta \bar{\varphi}_{0} / 2\right)\right)-\sin \left(m\left(\bar{\varphi}_{0}-\Delta \bar{\varphi}_{0} / 2\right)\right)\right] ; & m \geq 1,
\end{array}\right.
\end{aligned}
$$

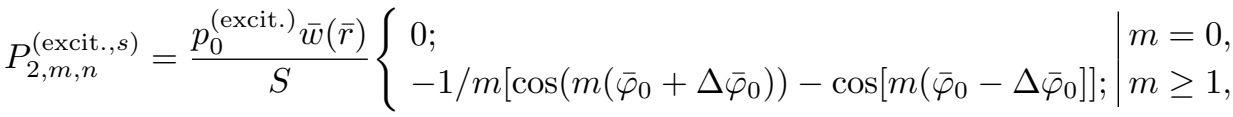

where

$$
\bar{w}(\bar{r})=\int_{\bar{r}-\Delta \bar{r} / 2}^{\bar{r}+\Delta \bar{r} / 2} W_{m, n}(r) r \mathrm{~d} r .
$$

The numerical calculations have been performed assuming that the plate is made of steel and the medium is air. The values of parameters describing the analyzed acoustic system have been presented in Table. The acoustic pressure has been calculated based on the formula from Eq. (4). However, the infinite series appearing in the formula have been limited to a finite number of terms to perform any numerical calculations. It means including only some vibrating modes and causes that an error occurs. All modes of the eigenfrequencies lower than the excitation frequency and some modes with the higher eigenfrequencies have to be included to provide the correct numerical results. The number of modes which have to be included in the numerical calculations will be indicated later for the assumed vibrating frequency. The 
TABLE

The values of the parameter describing the analyzed vibroacoustic system. The plate is made of steel and the medium is air.

\begin{tabular}{l|l}
\hline \hline \multicolumn{1}{c|}{ Parameter } & \multicolumn{1}{c}{ Value } \\
\hline plate radius & $a=0.2 \mathrm{~m}$ \\
sound velocity in the medium & $c=340 \mathrm{~m} / \mathrm{s}$ \\
Young's modulus & $E=205 \times 10^{9} \mathrm{~Pa}$ \\
plate thickness & $h=1 \times 10^{-3} \mathrm{~m}$ \\
plate's internal attenuation factor & $\bar{\eta}=10^{-4} \mathrm{~s}$ \\
Poisson's ratio & $\nu=0.3$ \\
plate density & $\rho=7700 \mathrm{~kg} / \mathrm{m}^{3}$ \\
medium density & $\rho_{0}=1.293 \mathrm{~kg} / \mathrm{m}^{3}$
\end{tabular}

numerical analysis of the acoustic pressure amplitude has been performed. The normalized value of the quantity, defined as

$$
D=|p(\boldsymbol{r})| / p_{\text {ref. }},
$$

where $p_{\text {ref. }}$ is the normalizing value, has been presented graphically.

The sound pressure level corresponding to the normalizing value equals $L_{\text {ref. }}=20 \log _{10}\left(p_{\text {ref. }} / p_{0}\right)$ where $p_{0}=20 \mu \mathrm{Pa}$ and has been included in the descriptions of each figure. The values of the acoustic pressure amplitudes have been calculated at large distance from the origin of Cartesian coordinate system using the obtained asymptotic formulae. It has been assumed that the excitation frequency does not exceed $f=400 \mathrm{~Hz}$. The minimum number of the included modes can be determined by means of an analysis of the relative error $E_{\text {trunc. }}$ resulting from the truncation in the series from Eq. (4). The error $E_{\text {trunc. }}$ has been calculated based on the following formula:

$$
E_{\text {trunc. }}=\frac{\left|D_{2 M+1,2 N}-D_{M, N}\right|}{D_{M, N}},
$$

where $D_{M, N}$ is the value obtained by including all modes $(m, n)$ where $m \leq M$ and $n \leq N$. The numbers $M, N$ determine, for the included in numerical calculations modes, the maximum numbers of the nodal diameters $m$ and the nodal circles $n$, respectively. Taking into account that the error $E_{\text {trunc. }}$ grows together with the vibration frequency, the calculations has been performed for the maximum frequency $f=400 \mathrm{~Hz}$. The different numbers $M, N$ have been analyzed. The acoustic attenuation has been neglected and the constants $c_{m, n}^{(c)}, c_{m, n}^{(s)}$ have been obtained from Eq. (12). In this case, it is not necessary to calculate the modal coefficients of acoustic impedance from the integral formulae and the computational complexity is reduced. The acoustic pressure can be obtained for a large number of the included vibrating modes.

The normalized amplitude of the acoustic pressure $D$ and the relative error $E_{\text {trunc. }}$ from Eq. (24) as the func-

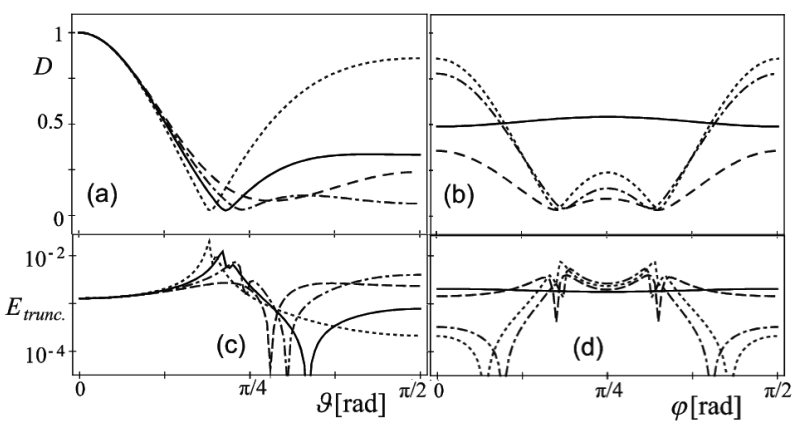

Fig. 3. The normalized acoustic pressure amplitude $D$ of the vibrating circular plate and the relative error $E_{\text {trunc. defined by Eq. (24). The excitation is given }}$ by Eq. (19a) and $\bar{r}_{0}=0.5 a, \bar{\varphi}_{0}=\pi / 4, F_{0}=1 \mathrm{~N}$, $f=400 \mathrm{~Hz}$. It has been assumed that: $l_{x} / a, l_{y} / a=1.5$, $r=16 \mathrm{~m}, L_{\text {ref. }}=55.8 \mathrm{~dB}$. Key for (a) and (c): solid line $-\varphi=\pi / 8$, dashed $-\varphi=\pi / 4$, dashed-dotted $\varphi=\pi / 3$, dotted $-\varphi=\pi / 2$; key for (b) and (d): solid line $-\vartheta=\pi / 8$, dashed $-\vartheta=\pi / 4$, dashed-dotted $\vartheta=3 \pi / 8$, dotted $-\vartheta=\pi / 2$.

tions of the spatial coordinates $\vartheta, \varphi$ have been shown in Fig. 3. The error has been calculated for $M=4$, $N=5$. The normalizing value $p_{\text {ref. }}$ is equal to the acoustic pressure amplitude in the case when $\vartheta=0$. The acoustic pressure has been calculated for a big distance $r=16 \mathrm{~m}$ from the origin of Cartesian coordinates system. Figure 3 shows that the relative error $E_{\text {trunc. }}$ does not exceed $1 \%$ for almost all analyzed field points. The exceptions appear near the points $[\vartheta, \varphi]=[\pi / 5, \pi / 8]$ and $[\pi / 5, \pi / 2]$. These large values of the error result from the small value of the acoustic pressure amplitude within the considered region of the acoustic field. It means that the number of the included vibrating modes is sufficient to analyze the sound radiation of investigated plate. In the case when the higher accuracy is needed, it is necessary to include more vibrating modes or analyze the lower vibration frequency.

Further numerical calculations have been performed for all modes $(m, n)$ where $m=0 \ldots 4$ and $n=1 \ldots 5$. The acoustic attenuation including requires the calculations of the 2025 modal coefficients of acoustic impedance based on the integral formulae (A.10) and obtaining the constants $c_{m, n}^{(c)}, c_{m, n}^{(s)}$ from Eqs. (A.8). The presented formulae describe the acoustic pressure within the Fraunhofer zone and enable to include the acoustic attenuation. The calculations performed in the case when the acoustic attenuation is included are much more time-consuming than the calculations performed when this factor is neglected. Therefore, it is important from practical viewpoint to determine the conditions when the neglecting of the acoustic attenuation does not cause a large error. For this purpose, the relative error $E_{\text {atten. }}$ resulting from the negligence of the acoustic attenuation has been analyzed. This error has been defined as 


$$
E_{\text {atten. }}=\frac{\left|D_{\text {atten. }}-D\right|}{D_{\text {atten. }}},
$$

where $D_{\text {atten. }}$ is the value calculated including the acoustic attenuation, $D$ denotes the approximate value obtained neglecting the acoustic attenuation. Obviously, the influence of the acoustic attenuation significantly depends on the vibration frequency. Therefore, the dependence of the relative error $E_{\text {atten. }}$ on the vibration frequency has been investigated. In Fig. 4a, the dashed line presents the quantity $D$ as a function of the frequency in the case when the acoustic attenuation has been neglected. The acoustic pressure has been calculated including the acoustic attenuation for some sample frequencies.
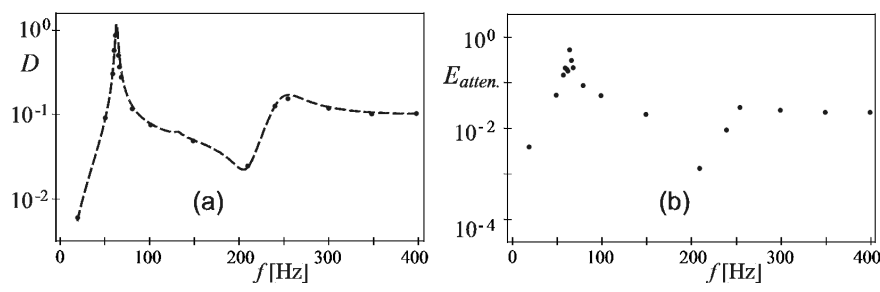

Fig. 4. The normalized acoustic pressure amplitude $D$ of the vibrating circular plate and the relative error

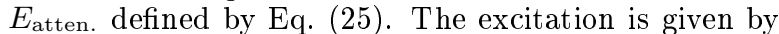
Eq. (19a) and $\bar{r}_{0}=0.5 a, \bar{\varphi}_{0}=\pi / 4$ and $F_{0}=1 \mathrm{~N}$. It has been assumed that the field point is located above the plate's centre at the distance of $H=16 \mathrm{~m}$ and $l_{x} / a, l_{y} / a=1.5, L_{\text {ref. }}=75.61 \mathrm{~dB}$. Key for (a): dashed line - the acoustic attenuation neglected, single points - the acoustic attenuation included; (b) points - error.

The results of these calculations have also been illustrated in Fig. 4a for comparison. The error $E_{\text {atten. as }}$ a function of the frequency has been shown in Fig. 4b. The amplitude of the acoustic pressure has been calculated at the field point located above the plate's centre at the distance of $H=16 \mathrm{~m}$. The normalizing value $p_{\text {ref. }}$. is equal to the maximum value of the acoustic pressure amplitude obtained for the analyzed frequencies and in the case when the acoustic attenuation is included.

The analysis of Fig. 4 shows that the acoustic attenuation significantly influences the value of the acoustic pressure amplitude. The error $E_{\text {atten. }}$ is greater than $1 \%$ for almost all investigated frequencies. This quantity achieves the greatest values for the frequencies near the resonance frequency $f=63.5 \mathrm{~Hz}$ corresponding to the eigenfrequency of the mode $(0,1)$. The maximum value of the error $E_{\text {atten. is equal to }} 44 \%$ for $65 \mathrm{~Hz}$. The acoustic attenuation shifts the resonance frequency towards the lower frequencies. This effect is particularly noticeable in the case of the first resonance frequency (Fig. 4a). Generally, the large values of the error $E_{\text {atten. }}$ occur for the resonance frequencies. It means that the acoustic attenuation can only be neglected for frequencies different from the frequencies near the resonance frequencies and when a high accuracy is not required.
The vertical baffles influence on the quantity $D$ has been investigated for the field point located above the plate's centre at the distance of $H=16 \mathrm{~m}$. It has been assumed that $f=250 \mathrm{~Hz}$ and the acoustic attenuation has been neglected.

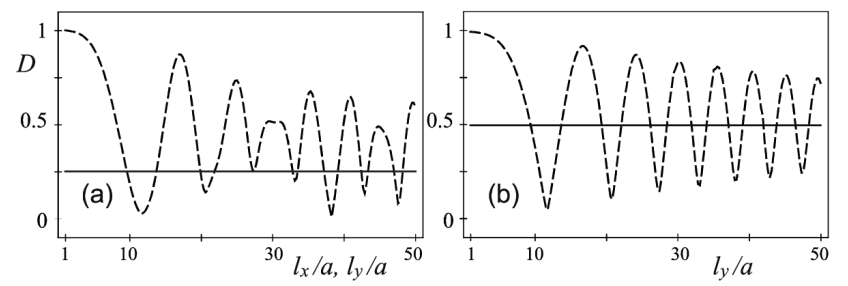

Fig. 5. The normalized acoustic pressure amplitude $D$ of the vibrating circular plate. The excitation is given by Eq. (19a) and $\bar{r}_{0}=0.5 a, \bar{\varphi}_{0}=\pi / 4, F_{0}=1 \mathrm{~N}$, $f=250 \mathrm{~Hz}$. It has been assumed that the field point is located above the plate's centre at the distance of $H=16 \mathrm{~m}, L_{\text {ref. }}=60 \mathrm{~dB}$ and for (b) $l_{x} / a=1.5$. Key - solid line: (a) without vertical baffles; (b) only one vertical baffle located on the plane $x z$.

In the case of Fig. 5, the normalizing value $p_{\text {ref. }}$ is equal to the amplitude of acoustic pressure radiated when the plate is located as close as possible to the vertical baffles i.e. when $l_{x}, l_{y}=a$. Assuming that $l_{x}=l_{y}$, the normalized amplitude of the acoustic pressure $D$ as a function of the distance from the vertical baffles has been presented in Fig. 5a. The dependence of the quantity $D$ on the distance $l_{y}$ has been shown in Fig. $5 \mathrm{~b}$ for $l_{x}=1.5 a$. Additionally, the solid line presents: in Fig. 5a - the quantity $D$ in the case when there are no vertical baffles, in Fig. $5 \mathrm{~b}$ - the quantity $D$ when only one vertical baffle exists. Based on Fig. 5a, b, it is possible to analyze the amplitudes' values of the acoustic pressure radiated in three specific cases, namely when: there are the two vertical baffles, only one vertical baffle exists, and there are no vertical baffles.

The plate located nearly the vertical baffles of the three-wall corner region radiate the acoustic pressure of the amplitude almost twice higher than the amplitude of the acoustic pressure radiated in the case when there is only one vertical baffle located near sound source and almost fourfold higher than the amplitude of the acoustic pressure radiated in the case when there are no vertical baffles. It means the significant increase in the acoustic pressure amplitude caused by the vertical baffles which reflect the acoustic waves. The same effect has also been observed in the case of the simpler circular sound sources such as pistons and membranes located near the vertical baffles [20, 22]. In the case of the analyzed plate, the vertical baffles cause the increase in the acoustic pressure for some plate's locations (maxima) and its reduction in other locations (nodes) (Fig. 5a, b). This results from the superposition of the direct and reflected acoustic waves.

The analysis of the sound radiation in the case of the acoustic sources located on the boundary of the two or three-wall corner regions enable concluding that the de- 
pendence of the acoustic pressure amplitude on the distance from the vertical baffles are similar for the vibrating pistons, membranes as well as for the investigated plate $[20,22]$. It means that the influence of the vertical baffles on the sound radiation is similar for the different circular acoustic sources. The analyzed situation, when the distances between the plate and the vertical baffles increase for the fixed vibration frequency, is analogous to the situation when the vibration frequency increases for the fixed plate's location. The distances between the plate and the vertical baffles related to the acoustic wavelength increases together with the vibration frequency. The frequency increase influences the acoustic pressure amplitude in the similar way as increase in the distances between plate and vertical baffles. The local maxima, analogous to those shown in Fig. 5, decrease when the vibration frequency increases. It should be noted that only the low audible frequencies i.e. from $20 \mathrm{~Hz}$ to $400 \mathrm{~Hz}$ have been considered in this study. Therefore, the attenuation of some acoustic waves in air has been neglected. However, this factor is very important for high frequencies.

In study [23], the total sound power has been analyzed in the case of the point excitation from Eq. (19a) and the excitation applied to the plate's segment defined by Eq. (19b). The sound source was the clamped circular plate located on one of the baffles of three-wall corner region. The values of the total sound power obtained in the case of both excitations has been compared with each other assuming that the following relation is satisfied (compare the excitation from Eq. (19a)):

$$
F_{0}=\int_{S} p_{2}^{\text {(excit.) }}\left(r_{0}, \varphi_{0}\right) \mathrm{d} S=p_{0}^{\text {(excit.) }} S_{0} .
$$

It has been proved in study [23] that the total power radiated in the case of the excitation defined by Eq. (19b) can be approximated by the total power radiated in the case of the point excitation from Eq. (19a). This approximation can be applied only for the low frequencies and when the area of the excited plate's segment is small compared with the whole plate's area.

The question arises whether the acoustic pressure distribution radiated in the case of the excitation from Eq. (19b) can be approximated by the acoustic pressure distribution radiated in the case of the excitation from Eq. (19a). The answer to the question can be found by analyzing the value of the relative error $E_{\text {excit. }}$ :

$$
E_{\text {excit. }}=\frac{\left|D_{2}-D_{1}\right|}{D_{2}}
$$

where $D_{1}$ is the quantity $D$ obtained for excitation from Eq. (19a), $D_{2}$ denotes the quantity $D$ obtained for excitation from Eq. (19b). The defined error results from the approximation of the excitation from Eq. (19b) by the excitation from Eq. (19a). It has been assumed that $S_{0} / S=0.001$ and $f=200 \mathrm{~Hz}$. The amplitude of the concentrated force $F_{0}$ defining the point excitation from Eq. (19a) has been determined based on the relation (26).

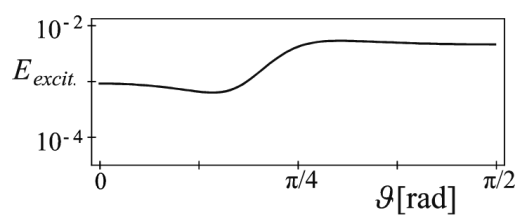

Fig. 6. The relative error $E_{\text {excit. }}$ defined by Eq. (27). The parameters describing the excitations given by Eqs. (19) are equal to $\bar{r}_{0}=0.5 a, \bar{\varphi}_{0}=\pi / 4, f=200 \mathrm{~Hz}$, $S_{0} / S=0.001$. It has been assumed that $r=16 \mathrm{~m}$, and $\varphi=\pi / 4$.

The amplitude of the excitation pressure $p_{0}^{\text {(excit.) }}$ does not influence the value of the relative error $E_{\text {excit. }}$ and has been chosen arbitrarily only to perform the numerical calculations. The dependence of the error $E_{\text {excit. }}$ on the spatial variables $\vartheta$ and $\varphi$ has been investigated. The calculations have been performed assuming that the distance between the analyzed field points and the origin of the global coordinate system is equal to $r=16 \mathrm{~m}$. The numerical analysis of the error $E_{\text {excit. }}$ enables concluding that the dependence of this quantity on the spatial variable $\vartheta$ is similar for $\varphi=\pi / 8, \pi / 4, \pi / 3, \pi / 2$. Therefore, the error $E_{\text {excit. }}$ as a function of the variable $\vartheta$ has been presented in Fig. 6 only in the case of $\varphi=\pi / 4$. The error $E_{\text {excit. }}$ does not exceed $0.7 \%$ for all the analyzed values of angle $\vartheta$ (cf. Fig. 6). The value of variable $\varphi$ does not significantly influence the error $E_{\text {excit. }}$ The curves presenting the error $E_{\text {excit. }}$ as a function of the angle $\varphi$ are almost the straight horizontal lines and they have not been illustrated. Based on the performed numerical analysis, it can be noted that for all values of the variable $\varphi$ the error $E_{\text {excit. }}$ does not exceed $0.1 \%$ when $\vartheta=\pi / 8$ and is less than $0.5 \%$ for $\vartheta=\pi / 4,3 \pi / 8, \pi / 2$. Taking into account that the error $E_{\text {excit. }}$ achieves the small value within the whole analyzed region, the acoustic pressure distribution obtained in the case of the excitation from Eq. (19b) can be approximated by means of the acoustic pressure distribution obtained in the case of the excitation defined by Eq. (19a). Consequently, the simpler mathematical model of excitation can be used which enable simplifying the numerical calculations.

\section{Conclusions}

The presented formulae of the acoustic pressure have the elementary form. They allow performing some fast numerical calculations within the Fraunhofer zone of three-wall corner region even for a large number of vibrating modes included. It is possible only when the acoustic attenuation is neglected. Otherwise, a large number of the modal coefficients of acoustic impedance have to be calculated numerically which causes that the number of the included vibrating modes is limited by the computational complexity. The obtained formulae enable reducing the time-consuming of the numerical calculations also in that case.

The performed analysis allows concluding that the relative error resulting from the negligence of the acoustic 
attenuation does not exceed a few percent. Only one exception appears within the narrow frequency range around the first resonance frequency where the error achieves large values. It has been noted that the influence of the vertical baffles on the sound radiation is significant. This influence is particularly noticeable when the source is located in the vicinity of the baffles. Adding one vertical baffle to the horizontal baffle with the vibrating source causes almost the twice increase in the acoustic pressure amplitude, whereas adding the two vertical baffles causes nearly the fourfold increase in this quantity. The results obtained are similar to those presented in previous studies and they are related to the sound radiation of the circular sound sources such as membranes and pistons. The theoretical and numerical analysis performed allows concluding that the excitation applied to the plate's segment of small area can be modelled by the point excitation.

\section{Appendix. The solution of the equation of motion for the excited plate}

The solution of the equation of motion given by Eq. (1) can be presented as the following double infinite series [23, 27-29]:

$$
\begin{aligned}
& v\left(r_{0}, \varphi_{0}\right)=-\mathrm{i} \omega \sum_{m=0}^{\infty} \sum_{n=1}^{\infty}\left[c_{m, n}^{(c)} W_{m, n}^{(c)}\left(r_{0}, \varphi_{0}\right)\right. \\
& \left.\quad+c_{m, n}^{(s)} W_{m, n}^{(s)}\left(r_{0}, \varphi_{0}\right)\right]
\end{aligned}
$$

where $W_{m, n}^{(c)}\left(r_{0}, \varphi_{0}\right)$ and $W_{m, n}^{(s)}\left(r_{0}, \varphi_{0}\right)$ for $m=0,1,2, \ldots$ and $n=1,2,3, \ldots$ constitute the complete system of the eigenfunctions, $c_{m, n}^{(c)}, c_{m, n}^{(s)} \in \mathbf{C}$ are unknown constants. The eigenfunctions of a clamped circular plate can be expressed in the form of

$$
\begin{aligned}
& \left\{\begin{array}{c}
W_{m, n}^{(c)}\left(r_{0}, \varphi_{0}\right) \\
W_{m, n}^{(s)}\left(r_{0}, \varphi_{0}\right)
\end{array}\right\}=W_{m, n}\left(r_{0}\right)\left\{\begin{array}{c}
\cos m \varphi_{0} \\
\sin m \varphi_{0}
\end{array}\right\}, \\
& W_{m, n}\left(r_{0}\right)=\frac{\sqrt{\varepsilon_{m}}}{\sqrt{2} J_{m}\left(\beta_{m, n}\right)} \\
& \quad \times\left[J_{m}\left(k_{m, n} r_{0}\right)-\frac{J_{m}\left(\beta_{m, n}\right)}{I_{m}\left(\beta_{m, n}\right)} I_{m}\left(k_{m, n} r_{0}\right)\right],
\end{aligned}
$$

where $J_{m}(\cdot), I_{m}(\cdot)$ are the $m$-th order Bessel function and the modified Bessel function, respectively, $\varepsilon_{m}=1$ for $m=0, \varepsilon_{m}=2$ for $m>0, k_{m, n}^{2}=\omega_{m, n} \sqrt{\rho h / D_{E}}, \omega_{m, n}$ is the eigenfrequency of the mode $(m, n), \beta_{m, n}=k_{m, n}$ a denotes the eigenvalue which is a solution of the frequency equation $J_{m+1}\left(\beta_{m, n}\right) I_{m}\left(\beta_{m, n}\right)+J_{m}\left(\beta_{m, n}\right) I_{m+1}\left(\beta_{m, n}\right)=$ 0 [27]. The constants $c_{m, n}^{(c)}, c_{m, n}^{(s)}$ appearing in Eq. (A.1) have to be calculated to obtain the solution for the equation of motion. These constants depend on: the external excitation, the distances between the plate centre and the vertical baffles, the vibration frequency and the internal attenuation. The eigenfunctions given by Eq. (A.2) satisfy the following relation [24]:

$$
\begin{aligned}
& \int_{0}^{a} \int_{0}^{2 \pi}\left\{\begin{array}{l}
W_{m, n}^{(c)}\left(r_{0}, \varphi_{0}\right) \\
W_{m, n}^{(s)}\left(r_{0}, \varphi_{0}\right)
\end{array}\right\}\left\{\begin{array}{l}
W_{k, l}^{(c)}\left(r_{0}, \varphi_{0}\right) \\
W_{k, l}^{(s)}\left(r_{0}, \varphi_{0}\right)
\end{array}\right\} r_{0} \mathrm{~d} r_{0} \mathrm{~d} \varphi_{0} \\
& =S \delta_{m, k} \delta_{n, l},
\end{aligned}
$$

where $S=\pi a^{2}$ is the plate's area, and $\delta_{m, n}$ denotes the Kronecker delta.

Inserting solution (A.1) into Eq. (1) and taking into account that $\nabla^{4} W_{m, n}^{(c)}\left(r_{0}, \varphi_{0}\right)=k_{m, n}^{4} W_{m, n}^{(c)}\left(r_{0}, \varphi_{0}\right)$, $\nabla^{4} W_{m, n}^{(s)}\left(r_{0}, \varphi_{0}\right)=k_{m, n}^{4} W_{m, n}^{(s)}\left(r_{0}, \varphi_{0}\right)$, leads to the following equations system [7, 23]:

$$
\begin{aligned}
& \left\{\begin{array}{c}
c_{m, n}^{(c)} \\
c_{m, n}^{(s)}
\end{array}\right\}\left(\frac{k_{m, n}^{4}}{k_{T}^{4}}-1\right)+\frac{1}{\rho h \omega^{2}}\left\{\begin{array}{c}
P_{m, n}^{(c)} \\
P_{m, n}^{(s)}
\end{array}\right\} \\
& =\frac{1}{\rho h \omega^{2}}\left\{\begin{array}{l}
P_{m, n}^{(\text {excit. }, c)} \\
P_{m, n}^{(\text {excit. }, s)}
\end{array}\right\},
\end{aligned}
$$

where

$$
\begin{aligned}
& \left\{\begin{array}{l}
P_{m, n}^{(c)} \\
P_{m, n}^{(s)}
\end{array}\right\}=\frac{1}{S} \int_{S} p\left(r_{0}, \varphi_{0}\right)\left\{\begin{array}{l}
W_{m, n}^{(c)}\left(r_{0}, \varphi_{0}\right) \\
W_{m, n}^{(s)}\left(r_{0}, \varphi_{0}\right)
\end{array}\right\} \mathrm{d} S \\
& \left\{\begin{array}{l}
P_{m, n}^{\text {(excit. }, c)} \\
P_{m, n}^{(\text {excit. }, s)}
\end{array}\right\}=\frac{1}{S} \int_{S} p^{(\text {excit. })}\left(r_{0}, \varphi_{0}\right) \\
& \quad \times\left\{\begin{array}{l}
W_{m, n}^{(c)}\left(r_{0}, \varphi_{0}\right) \\
W_{m, n}^{(s)}\left(r_{0}, \varphi_{0}\right)
\end{array}\right\} \mathrm{d} S,
\end{aligned}
$$

After employing Eq. (4), the equations system (A.5) can be written by means of the modal coefficients of the acoustic impedance (cf. [23]):

$$
\begin{aligned}
& \left\{\begin{array}{l}
c_{m, n}^{(c)} \\
c_{m, n}^{(s)}
\end{array}\right\}\left(\frac{k_{m, n}^{4}}{k_{T}^{4}}-1\right)-\mathrm{i} \varepsilon_{0} \frac{\omega_{0,1}}{\omega} \\
& \quad \times \sum_{k=0}^{\infty} \sum_{l=1}^{\infty}\left(c_{k, l}^{(c)}\left\{\begin{array}{l}
\zeta_{k, c)}^{(c, l)} \\
\zeta_{k, l ; m, n}^{(c), ~}
\end{array}\right\}+c_{k, l}^{(s)}\left\{\begin{array}{l}
\zeta_{k, l, m, m, n}^{(s, c)} \\
\zeta_{k, l ; m, n}^{(s, s)}
\end{array}\right\}\right) \\
& =\frac{1}{\rho h \omega^{2}}\left\{\begin{array}{c}
P_{m, n}^{(\text {excit. }, c)} \\
P_{m, n}^{(\text {excit. }, s)}
\end{array}\right\},
\end{aligned}
$$

where

$$
\begin{aligned}
& \zeta_{m, n ; k, l}^{(i, j)}=\frac{\mathrm{i}}{S \rho_{0} c \omega_{m, n}} \int_{S^{\prime}} p_{m, n}^{(i)}(\boldsymbol{r}) W_{k, l}^{(j)}(\boldsymbol{r}) \mathrm{d} S^{\prime}, \\
& \quad i, j \in\{c, s\}
\end{aligned}
$$

are the modal coefficients of the acoustic impedance, $\boldsymbol{r}=[x, y, 0]$ and $\varepsilon_{0}=\rho_{0} c /\left(\rho h \omega_{0,1}\right)$ is the coefficient of the acoustic attenuation.

The modal coefficients of the acoustic impedance have been obtained using the Green function in the Fourier representation. Finally, the following integral formulae have been formulated [23]

$$
\zeta_{m, n ; k, l}^{(i, j)}=4 \beta^{2} \sqrt{\varepsilon_{m} \varepsilon_{k}} \int_{0}^{\infty} \frac{\phi_{m, k}^{(i, j)}(\tau) \psi_{m, n}(\tau) \psi_{k, l}(\tau) \tau \mathrm{d} \tau}{\sqrt{1-\tau^{2}}},
$$




$$
\begin{aligned}
& \left\{\begin{array}{l}
\phi_{m, k}^{(c, c)}(\tau) \\
\phi_{m, k}^{(s, s)}(\tau)
\end{array}\right\}=(-1)^{k+m}\left[ \pm J_{k+m}(2 \beta L \tau)\right. \\
& \left.\quad \times \cos \left((k+m) \varphi_{L}\right)+J_{k+m}\left(2 \beta L_{x} \tau\right)\right] \\
& \quad+(-1)^{k}\left[J_{k-m}(2 \beta L \tau) \cos \left((k-m) \varphi_{L}\right)\right. \\
& \left.\quad \pm J_{k-m}\left(2 \beta L_{x} \tau\right)\right]+\cos ((k-m) \pi / 2)\left[J_{k+m}\left(2 \beta L_{y} \tau\right)\right. \\
& \left.\quad \pm J_{k-m}\left(2 \beta L_{y} \tau\right)\right]+\delta_{m, k}\left\{\begin{array}{l}
2 / \varepsilon_{m} \\
\operatorname{sgn}(m)
\end{array}\right\}, \\
& \phi_{m, k}^{(c, s)}(\tau)=(-1)^{k+m} J_{k+m}(2 \beta L \tau) \sin \left((k+m) \varphi_{L}\right) \\
& +(-1)^{k} J_{k-m}(2 \beta L \tau) \sin \left((k-m) \varphi_{L}\right) \\
& \quad-\sin ((k-m) \pi / 2)\left[J_{k+m}\left(2 \beta L_{y} \tau\right)+J_{k-m}\left(2 \beta L_{y} \tau\right)\right],
\end{aligned}
$$

$$
\phi_{m, k}^{(s, c)}(\tau)=\phi_{k, m}^{(c, s)}(\tau),
$$

where $L=\sqrt{L_{x}^{2}+L_{y}^{2}}=|\boldsymbol{l}| / a>1, L_{x}=l_{x} / a>1$, $L_{y}=l_{y} / a>1, \varphi_{L}=\arctan \left(L_{y} / L_{x}\right)$ is the angle between the vector $l$ and the $x$-axis (see Fig. 1 ).

The acoustic attenuation can be neglected when $\varepsilon_{0} \rightarrow 0$. Assuming in equations system (A.8) $\varepsilon_{0}=0$ yields

$$
\left\{\begin{array}{c}
c_{m, n}^{(c)} \\
c_{m, n}^{(s)}
\end{array}\right\}=\frac{k_{T}^{4}}{\rho h \omega^{2}\left(k_{m, n}^{4}-k_{T}^{4}\right)}\left\{\begin{array}{l}
P_{m, n}^{(\text {excit. }, c)} \\
P_{m, n}^{(\text {excit. }, s)}
\end{array}\right\} .
$$

The above equations allow to obtain the necessary constants $c_{m, n}^{(c)}, c_{m, n}^{(s)}$ without calculating the modal coefficients of the acoustic impedance which are defined by the integral formulae. It reduces the numerical calculation time. However, employing Eq. (A.12) is possible only in special cases. Generally, it can cause some large errors, especially for the frequencies near the resonance frequencies.

\section{References}

[1] R.L. Pritchard, J. Acoust. Soc. Am 32, 730 (1960).

[2] E.M. Arase, J. Acoust. Soc. Am. 36, 1521 (1964).

[3] H. Levine, J. Sound Vibrat. 89, 447 (1983).
[4] A. Berry, J.L. Guyader, J. Nicolas, J. Acoust. Soc. Am. 86, 2792 (1990).

[5] M.R. Lee, R. Singh, J. Acoust. Soc. Am. 95, 3311 (1994).

[6] J.P. Arenas, Int. J. Occupat. Safety Ergon. 15, 401 (2009).

[7] H. Levine, F.G. Leppington, J. Sound Vibrat. 121, 269 (1988).

[8] D. Zou, M.J. Crocker, Archiv. Acoust. 34, 25 (2009).

[9] H. Lee, R. Singh, J. Sound Vibrat. 282, 313 (2005).

[10] J. Cieślik, J. Pieczara, Archiv. Acoust. 33 (4 Suppl.), 201 (2008).

[11] M. Yairi, K. Sakagami, Appl. Acoust. 63, 737 (2002).

[12] N. Hashimoto, Appl. Acoust. 62, 429 (2001).

[13] D. Zou, M.J. Crocker, Archiv. Acoust. 34, 13 (2009).

[14] M. Kozupa, J. Wiciak, Acta Phys. Pol. A 118, 95 (2010).

[15] L. Leniowska, Archiv. Acoust. 34, 507 (2009).

[16] M. Pawełczyk, Archiv. Acoust. 33, 509 (2008).

[17] W. Batko, M. Kozupa, Archiv. Acoust. 33(S4), 195 (2008).

[18] S.M. Hasheminejad, M. Azarpeyvand, Shock Vibrat. 11, 625 (2004).

[19] W.P. Rdzanek, K. Szemela, Archiv. Acoust. 32, 339 (2007).

[20] W.P. Rdzanek, K. Szemela, D. Pieczonka, Archiv. Acoust. 32, 883 (2007).

[21] W.P. Rdzanek, W.J. Rdzanek, K. Szemela, Archiv. Acoust. 34, 75 (2009).

[22] W.P. Rdzanek, K. Szemela, D. Pieczonka, Archiv. Acoust. 36, 121 (2011).

[23] K. Szemela, W. Rdzanek, D. Pieczonka, Acta Phys. Pol. A 119, 1050 (2011).

[24] L. Meirovitch, Analytical Methods in Vibrations, Macmillan, New York 1967.

[25] P.M. Morse, K.U. Ingard, Theoretical Acoustics, McGraw-Hill, Princeton 1968.

[26] H. Weyl, Ann. Phys. 60, 481 (1919).

[27] N.W. McLachlan, Bessel Functions for Engineers, Clarendon Press, Oxford 1955.

[28] A.W. Leissa, Vibration of Plates, Vol. SP-160, NASA, U.S. Government Printing Office, Washington 1969.

[29] E. Skudrzyk, Simple and Complex Vibration Systems, Pennsylvania State University Press, London 1968. 